\title{
Osteoporose - A Importância da Prevenção de Quedas ${ }^{(*)}$
}

\section{Osteoporosis - The Importance of Preventing Falls}

\author{
Rachel Riera $^{(1)}$, Virgínia F. Moça Trevisani ${ }^{(2)}$ e João Paulo Nogueira Ribeiro ${ }^{(3)}$
}

\section{RESUMO}

O ritmo de crescimento da população com 60 anos ou mais têm sido intenso nos últimos 30 anos em nosso país. Esta mudança demográfica leva à transição epidemiológica, caracterizada pela queda da mortalidade infantil, redução das doenças infecciosas e aumento das doenças crônico-degenerativas (como a osteoporose). O aumento da morbimortalidade pela osteoporose está associado a custos econômicos significativos relacionados à hospitalização, cuidados ambulatoriais, institucionalização, incapacidades e mortes prematuras.

Sendo a fratura a conseqüência clínica da osteoporose, a avaliação do ambiente domiciliar para identificar e eliminar riscos ambientais tem grande validade como estratégia de prevenção de quedas, já que estas são responsáveis por mais de $85 \%$ das fraturas.

Desta forma, cumpre ressaltar que quedas são eventos freqüentes e de alta morbidade em idosos que vivem na comunidade.

Os desafios para o reumatologista e outros profissionais da saúde estão na identificação precoce dos fatores de risco para a osteoporose, na educação dos pacientes e na intervenção ao longo de toda a vida, tanto em homens quanto em mulheres, prática ainda timidamente desenvolvida em nosso meio.

Palavras-chave: osteoporose, queda, fratura, prevenção.

\section{INTRODUÇÃO}

O ritmo de crescimento da população com 60 anos ou mais têm sido intenso nos últimos 30 anos no nosso país. Esta mudança demográfica leva à transição epidemiológica, caracterizada pela queda da mortalidade infantil, redução das doenças infecciosas e aumento das doenças crônicodegenerativas, como a osteoporose e conseqüentemente de sua manifestação clínica, a fratura ${ }^{(1)}$.

\begin{abstract}
The population growth for individuals over 60 years old has been intensified in the past thirty years in our country. This demographic change leads to epidemiological transition, characterized by the decrease of childhood death rate, reduction of infectious diseases and increase of chronic degenerate disease, such as osteoporosis. The mortality and morbidity increase due to osteoporosis has been associated with substantial economic costs attributed to hospitalization, admission to an institution, disabilities and premature deaths.

Fracture is the clinical consequence of osteoporosis. Then, the evaluation of the environment at home in order to identify and remove hazards is very important as prevention strategy for falls, since they are the leading cause of more than $85 \%$ of fractures. So, it is important to remember that falls are common and considered as high morbidity events in the elderly living in communities.

The challenge to the rheumatology clinician and other health professionals is the early recognition of risk factors for osteoporosis and falling; the education of patients and the intervention during the whole life, for both, men and women, is still a conduct not much developed in our community.
\end{abstract}

Keywords: osteoporosis, falls, fractures, prevention.

O aumento da mortalidade e morbidade pela osteoporose está associado a custos econômicos significativos relacionados à hospitalização, cuidados ambulatoriais, institucionalização, incapacidades e mortes prematuras ${ }^{(2)}$.

No Brasil, dispomos de poucos trabalhos sobre a ocorrência de faturas osteoporóticas. Nos EUA, os gastos excedem a 10 bilhões de dólares com os pacientes que sofreram fratura de fềmur e mais 18 bilhões são gastos com as outras fraturas. Dadas as tendências demográficas, prevê-se que, em 2020, o

\footnotetext{
* Trabalho realizado pela Pós-graduação de Medicina Interna e Terapêutica da Universidade Federal de São Paulo. Recebido em 3/12/2002. Aprovado, após revisão, em 20/08/2003.

1. Pós-graduanda de Medicina Interna e Terapêutica da Universidade Federal de São Paulo.

2. Professora Dra. Chefe da Disciplina de Reumatologia da Faculdade de Medicina de Santo Amaro, Professora Assistente da Disciplina de Medicina de Urgência da Universidade Federal de São Paulo

3. Pós-graduando de Medicina Interna e Terapêutica da Universidade Federal de São Paulo.

Endereço para correspondência: Departamento de Medicina - Pós-graduação de Medicina Interna e Terapêutica da Universidade Federal de São Paulo. Rua Pedro de Toledo, Vila Clementino, CEP 04024-002, São Paulo-SP. E-mail: rachelriera@hotmail.com
} 
tratamento das seqüelas da osteoporose custará de 30 a 60 bilhões de dólares/ano, daí a premência de se implantarem estratégias efetivas de prevenção e tratamento ${ }^{(2)}$.

\section{OSTEOPOROSE}

A osteoporose é uma doença sistêmica caracterizada por diminuição da massa óssea e deterioração da microarquitetura do tecido ósseo, com conseqüente aumento da fragilidade do osso e da susceptibilidade a fraturas ${ }^{(3)}$. Ela é definida pela medida da densidade mineral óssea (DMO), por meio da densitometria óssea, sendo diagnosticada quando o valor da densidade mineral óssea é de, pelo menos, 2,5 desvios padrão abaixo do valor médio encontrado em indivíduos jovens e saudáveis ${ }^{(4)}$.

O tecido ósseo tem como função primordial manter um bom estado anatômico e funcional do esqueleto e, conseqüentemente, a homeostase mineral ${ }^{(5)}$. A manutenção equilibrada deste sistema está apoiada basicamente em dois processos que ocorrem simultaneamente:

1) formação incessante do osteóide pelos osteoblastos, que, fixando cálcio e fosfato, constituem o osso jovem, compensando desta maneira suas perdas;

2) reabsorção do osso adulto pelos osteoclastos e osteócitos, fornecendo aos humores o cálcio e o fósforo necessários para a manutenção da homeostase mineral ${ }^{(5)}$.

Estes processos estão em equilíbrio constante, promovendo uma remodelação óssea contínua, sob a influência de fatores sistêmicos e locais. A perda deste equilíbrio leva ao aparecimento das doenças osteometabólicas, entre as quais a osteoporose representa $80 \%$ dos $\operatorname{casos}^{(5)}$.

A osteoporose pode ser classificada de acordo com a etiologia, como primária ou secundária, dependendo da existência ou não de uma condição clínica associada que sabidamente cause perda óssea ${ }^{(6)}$ (Tabela 1). A forma primária comum, denominada "osteoporose involutiva", começa na meia-idade, torna-se progressivamente mais comum com a idade avançada e é dividida em tipo I ou pós-menopausa e tipo II ou senil( ${ }^{(6)}$ (Tabela 2).

Em relação à epidemiologia, estudos que utilizaram a densidade mineral óssea do fềmur proximal mostraram que $13 \%$ a $18 \%$ das mulheres com mais de 50 anos têm osteoporose e 37\% a 50\% têm osteopenia; 3\% a 6\% dos homens na mesma faixa etária têm osteoporose e $28 \%$ a $47 \%$ têm osteopenia ${ }^{(4)}$. $\mathrm{Na}$ América do Sul o conhecimento da epidemiologia da osteoporose ainda é incompleto, com estudos realizados apenas em alguns países e com pequeno número de pacientes ${ }^{(4)}$. Estima-se que, após os 50 anos, 1 a cada 4 mulheres e 1 a cada 8 homens apresentem osteoporose ${ }^{(2)}$.

O quadro clínico é caracterizado por dorsalgia, perda da altura, deformidade da coluna (especialmente hipercifose) e fraturas das vértebras, do fêmur, dos punhos e de outros ossos ${ }^{(6)}$. Sua evolução é lenta durante anos, com

TABELA 1

Classificação das causas de osteoporose

Primária
juvenil, idiopática, involutiva
Dist. Medula Óssea
mieloma múltiplo e alterações correlatas, mastocitose sistêmica,
carcinoma disseminado
Endócrina
hipogonadismo, agenesia ovariana, excesso de glicocorticóide,
diabete (?) hipertireoidismo, hiperparatireoidismo
Doença Tecido Conjuntivo
osteogênese imperfeita, homocistinúria, síndromes de Marfan e
$\quad$ Ehlers-Danlos
Gastrintestinal
gastrectomia subtotal, alactasia, icterícia obstrutiva crônica,
cirrose biliar primária, desnutrição grave, síndrome de mal
absorção
Outras causas
imobilização, alcoolismo crônico, DPOC, artrite reumatóide (?),
uso crônico de heparina e de anticonvulsivantes (?)

TABELA 2

Osteoporose inVOLUTIVA

\begin{tabular}{lcc}
\hline & Tipo 1 - Pós-menopausa & Tipo 2 - Senil \\
\hline Idade & $55-70$ anos(5-15 anos de pós-menopausa) & acima 70 anos(20-40 anos de pós-menopausa) \\
Sexo & 20 mulheres / 1 homem & 3 mulheres / 1 homem \\
Etiologia & diminuição de estrogênio & hiperparatireoidismo secundário \\
Local da fratura & vértebra, radio distal & fêmur, vértebra, úmero \\
Tipo de osso & trabecular & cortical e trabecular \\
\hline
\end{tabular}


curtos surtos agudos, relacionados principalmente a colapsos vertebrais ${ }^{(5)}$.

Os fatores de risco para osteoporose são apresentados na Tabela $3^{(2)}$.

TABELA 3

FATORES DE Risco para osteoporose

Fatores maiores
Sexo feminino
Baixa massa óssea
Fratura prévia
Raça asiática ou caucasiana
Idade avançada em ambos os sexos
História materna de fratura de fêmur e/ou osteoporose
Menopausa precoce não tratada (antes dos 40 anos)
Tratamento com corticóides
Fatores menores
Amenorréia primária ou secundária
Hipogonadismo primário ou secundário em homens
Perda de peso após os 25 anos ou indíce de massa corpórea baixo
(menor que 19)
Tabagismo, alcoolismo, sedentarismo
Tratamento com drogas que induzem perda de massa óssea
(heparina, warfarina, fenobarbital, fenitoína, carbamazepina,
lítio e metotrexato)
Imobilização prolongada
Passado de dieta pobre em cálcio
Doenças que induzem a perda de massa óssea

\section{FRATURAS}

A conseqüência clínica da osteoporose é a fratura, que em mais de $85 \%$ dos casos é causada por quedas ${ }^{(1)}$. Os locais de maior ocorrência são vértebras, punhos e região proximal do fêmur ${ }^{(2)}$.

As fraturas vertebrais podem ocorrer sem sintomas, porém, à medida que aumentam em número e intensidade podem estar associadas a grau significativo de morbidade ${ }^{(4)}$. No entanto, apenas 30\% dos pacientes com este tipo de fratura procuram atendimento médico ${ }^{(2)}$. As atividades diárias do paciente, tais como vestir-se, caminhar, limpar a casa ou praticar esportes, podem ser seriamente comprometidas por dor e incapacidade conseqüentes à deformidade dorsolombar ${ }^{(4)}$.

As fraturas de quadril (região proximal do fêmur) são as mais devastadoras, em virtude da elevada morbimortalidade, e após o evento, aproximadamente $50 \%$ das pessoas se tornam incapazes de andar sem ajuda, 25\% necessitam de cuidados de longa duração e $10 \%$ a $20 \%$ morrem nos 6 meses seguintes à fratura ${ }^{(4,7)}$.

A identificação precoce dos fatores de risco é a principal meta na introdução de estratégias efetivas de prevenção da fratura em pacientes com osteoporose. As medidas preventivas podem ser farmacológicas e não-farmacológicas. Entre estas últimas, estão a nutrição adequada, os bons hábitos de vida e o controle do ambiente para a prevenção de quedas ${ }^{(2)}$.

\section{QUEDAS}

Queda é definida como sendo a ocorrência de um evento não intencional que leva uma pessoa inadvertidamente a cair ao chão em um mesmo nível ou em outro inferior ${ }^{(1)}$.Quedas associadas a perda de consciência ou ataque convulsivo súbito, decorrentes de um evento cérebro vascular agudo, de doença epilética, de grave acidente automobilístico, de atividade recreacional exagerada ou atos de violência fisica, geralmente são excluídas da definição de quedas em idosos ${ }^{(1)}$.

Quedas são eventos freqüentes e de alta morbidade em idosos que vivem na comunidade. A incidência estimada mundial anual é de 30\% para pessoas com 65 anos ou mais, $35 \%$ para aquelas com 70 anos ou mais, $32 \%$ a $42 \%$ para aquelas com 75 anos ou mais e $50 \%$ para aquelas com 80 anos ou mais ${ }^{(8-11)}$. Das pessoas que caem, 10\% apresentam sérios danos, como fratura, deslocamento de articulação ou ferimento craniano grave $e^{(10,12,13)}$. A taxa de mortalidade por 100.000 idosos, devido à queda, aumenta com a idade, sendo de 10,2 dos 65 aos 74 anos e de 147,0 dos 85 anos em diante ${ }^{(13)}$.

No Brasil, de acordo com dados do Ministério da Saúde (SIH/SUS), a taxa de mortalidade hospitalar por quedas, em fevereiro de 2000 , foi de $2,58 \%$, sendo que a região Sudeste apresentou a maior taxa $(3,71 \%)^{(1)}$.

As consequiências das quedas incluem: dor, perda da confiança e medo de quedas subseqüentes, decúbito de longa duração, restrição das atividades, declínio funcional e redução da independência ${ }^{(8,10,14)}$. Aproximadamente 50\% dos pacientes com queda seguida de fratura terão incapacidade funcional permanente maior do que tinham antes do evento ${ }^{(4)}$.

\section{CAUSAS DE QUEDAS}

Muitos fatores estão implicados como causadores e parece mais provável que combinações de fatores sejam mais

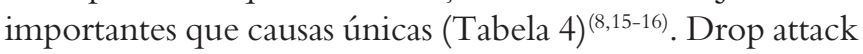
(ataque de queda) é uma causa de queda de etiologia pouco conhecida, caracterizado por uma queda sem perda da 
consciência, que não pode ser explicada por fraqueza muscular, distúrbios neurológicos focais ou arritmias e não é induzido por movimentos rotatórios da cabeça ou vertigem ou outra sensação cefálica. As teorias mais aceitas associam este fenômeno a anormalidades na função postural ou a uma disfunção transitória na formação reticular que controla a musculatura antigravitacional ${ }^{(8)}$.

Em relação às medicações, é difícil separar o seu efeito daquele das doenças para as quais são prescritas, mas é provável que o consumo de qualquer medicamento esteja associado a um risco aumentado de quedas ${ }^{(17)}$. Residentes de casa de repouso em uso de mais de quatro medicações parecem possuir um risco até três vezes maior de queda e as classes associadas a este aumento incluem drogas psicotrópicas, cardiovasculares, corticosteróides e antiiflamatórios não-hormonais ${ }^{(8)}$.

Os fatores extrínsecos incluem riscos ambientais, riscos nas atividades diárias e, em indivíduos mais frágeis, movimentos como se virar, inclinar-se ou se esticar para alcançar um objeto. Os riscos ambientais ocorrem em até 50\% das quedas não-sincopais, predominando em 30\% destas, e são descritos como prevalentes nos lares de idosos, independente do grau de incapacidade destes ou do tipo de $\operatorname{casa}^{(1,8,18-20)}$.

Os riscos no ambiente domiciliar incluem: escadas, piso irregular, superfícies lisas, tapetes soltos ou com bordas dobradas, iluminação inadequada, objetos em locais de passagem, assentos baixos (cadeira, cama, vaso sanitário), calçado inadequado, objetos fora de alcance, boxe e banheira conjugados e boxe sem barras de apoio(8,21). Um estudo de Campbell et al., em 1990, revelou que os objetos mais relacionados a quedas foram irregularidades do piso e presença de degraus ${ }^{(22)}$.

Gill et al., em 1999, mostraram os principais fatores de risco ambientais relacionados com algumas atividades diárias

TABELA 4

Causas de quedas

\begin{tabular}{l}
\hline Hipotensão postural \\
Diminuição da força muscular \\
Diminuição da circulação cerebral \\
Diminuição da condução do estímulo \\
Diminuição da visão-audição-equilíbrio \\
Ataques de quedas (drop attacks) \\
Uso de medicações \\
Riscos extrínsecos (riscos na atividade diária e riscos ambientais) \\
\hline
\end{tabular}

relevantes que podem ser amenizados com uma intervenção, reduzindo, assim, o risco de quedas ${ }^{(23)}$. Desta forma, a dificuldade de transferência (posições inicial e final com alturas diferentes) foi associada à presença de cadeira e vasos baixos ou cambaleantes; a dificuldade de equilíbrio, à ausência de barras de apoio no boxe do banheiro; a dificuldade de marcha, a tapetes soltos, carpetes com bordas enroladas ou desfiadas e passagem obstruída ${ }^{(23)}$.

A maior parte das quedas ocorre em hora de atividade máxima no dia, somente 19\% ocorrem à noite (entre as $21 \mathrm{~h} \mathrm{e} 7 \mathrm{~h}$ ) e nos meses de inverno e dias mais frios há um aumento da incidência de quedas e fraturas em mulheres ${ }^{(8,23)}$. Aproximadamente $65 \%$ das mulheres e $44 \%$ dos homens caem dentro de casa, e cerca de 11\% das mulheres e $25 \%$ dos homens, no jardim de suas residências. As quedas dentro de casa ocorrem nos cômodos mais utilizados: 21,4\% acontecem no quarto; $19,1 \%$, na cozinha e $27,4 \%$, na sala ${ }^{(8,23)}$.

\section{COMENTÁRIOS}

Os desafios para os profissionais de saúde estão na identificação dos fatores de risco para a osteoporose, na educação dos pacientes e na intervenção ao longo de toda a vida, tanto em homens quanto em mulheres, prática ainda timidamente desenvolvida em nosso meio ${ }^{(2)}$.

Dentro dessa linha, a avaliação do ambiente domiciliar para identificar e eliminar riscos ambientais tem grande validade como estratégia de prevenção de quedas ${ }^{(19)}$. Esta estratégia deve ser colocada em prática após confirmação dos fatores de risco associados a quedas, em pacientes com ou sem o diagnóstico de osteoporose.

Estudos sobre programas de visitas domiciliares envolvendo enfermeiras e outros agentes de saúde têm mostrado muitos benefícios, incluindo diminuição da mortalidade, redução da deterioração funcional e das admissões hospitalares e aumento da aderência a guidelines de prevenção ${ }^{(20)}$. A visita domiciliar permite ainda observar como o paciente está lidando com a doença e com a sua incapacidade ${ }^{(20)}$.

Deve ser ressaltada a importância da orientação do paciente, ainda nas consultas ambulatoriais, em relação aos fatores de risco presentes no seu domicílio. Há de se enfatizar diante do paciente que tais fatores podem ser eliminados facilmente e sem grandes despesas, mas que colaboram de modo imperativo para a manutenção de sua independência nas atividades diárias. 


\section{REFERÊNCIAS}

1. Brito FC, Costa SMN.Quedas. In: Netto MP, Brito FC. Urgências em Geriatria. Atheneu, 2001; 1.a ed, pp.323-35.

2. Pereira SRM, Mendonça LMC. Osteoporose e Osteomalácia. In: Freitas EV, Py L, Neri AL, Cançado FAX, Gorzoni ML, Rocha SM. Tratado de Geriatria e Gerontologia. Guanabara Koogan, 2002; 1.a ed, pp.515-31.

3. National Osteoporosis Foundation. 1996 and 2015 osteoporosis prevalence figures: state by state report. Washington: National Osteoporosis Foundation, 1997.

4. Zerbini CAF. Osteoporose: Uma revisão conceitual. Jovem Médico 2000; ed. especial. jun: 31-8.

5. Szejnfeld VL, Atra E. Osteoporose. In: Valle JR, Ramos OL, Rothschild HA (ed). Atualização Terapêutica. Artes Médicas, 2001; 20.a ed, pp.601-4.

6. Riggs BL. Osteoporose. In: Cecil - Tratado de Medicina Interna. Guanabara Koogan, 1993; 19.a ed, pp. 1456-61.

7. Kelley GA. Aerobic exercise and bone density at the hip in postmenopausal women: A Meta-Analysis.Prev Med 1998; 27:798-807.

8. Heckmann M, Junior CMP. Distúrbios da postura, marcha e quedas. In: Freitas EV, Py L, Neri AL, Cançado FAX, Gorzoni ML, Rocha SM. Tratado de Geriatria e Gerontologia. Guanabara Koogan, 2002;1a. ed, pp. 624-34.

9. Tinetti ME, Speechley M, Ginter SF. Risk factors for falls among elderly persons living in the community. N Engl J Med 1998; 319:1701-7.

10. O'Loughlin JL, Robitaille Y, Boivin JF, et al. Incidence of and risk factors for falls and injurious falls among the community-dwelling elderly. Am J Epidemiol 1993;137:342-54.
11. Blake AJ, Morgan K, Bendall MJ, et al. Falls by elderly people at home: Prevalence and Associated Factors. Age Ageing 1988;17:365-72.

12. Tinetti ME, DoucetteJ, Claus E, et al. Risk factors for serious injury during falls by older persons in the community. Am J Geriatr Soc 1995;43:1214-21.

13. Public Health Surveillance of 1990 injury control objectives for the nation. MMWR CDC Surveill Summ 1988;37:1-68.

14. Tinetti ME, Williams CS. Falls, injuries due to falls and the risk of admission to a nursing home. N Engl J Med 1997;337:1279-84.

15. Isaacs B. Clinical and laboratory studies of falls in old people: Prospects for prevention. Clin Geriatr Med 1985;1:513-24.

16. Macdonald JB. The role of drugs in falls in the elderly. Clin Geriatr Med 1985;1:621-36.

17. Monane M, Avorn J. Medications and falls. Clin Geriatr Med 1996;12:847-58.

18. Waller JA. Falls among the elderly - human and environmental factors. Accid Anal \& Prev 1978;10:21-33.

19. Gill TM, Williams CS, Tinetti ME. Environmental hazards and the risk of nonsyncopal falls in the homes of community-living older persons. Med Care 2000;38(12):1174-83.

20. Rubenstein LZ. The Importance of including the home environment in assessment of frail older people. J Am Geriatr Society 1999;47:111-12.

21. Archea JC. Environmental risk factor associated with stair accidents by the elderly. Clin Geriatr Med 1985;1:555-69.

22. Campbell AJ, Borrie J, Spears GF, et al. Circumstances and consequences of falls experienced by a community population 70 years and over during a prospective study. Age and Ageing 1990;19:136-41.

23. Gill TM, Robison JT, Williams CS, et al. Mismatches between the home environment and physical capabilities among communityliving older persons. J Am Geriatr Soc 1999;47:88-92. 